\title{
172 Simple enucleation versus standard partial nephrectomy for clinical T1 renal tumors: Intraoperative, early post-operative and pathological outcomes from a prospective multicenter comparative study (RECORd Project)
}

Eur Urol Suppl 2013;12;e172

Verze P. ${ }^{1}$, Fusco F. ${ }^{1}$, Minervini A. ${ }^{2}$, Antonelli A. ${ }^{3}$, Bianchi G. ${ }^{4}$, Bocciardi A. ${ }^{5}$, Cosciani Cunico S. ${ }^{3}$, Ficarra V. ${ }^{6}$, Fiori C. ${ }^{3}$, Giancane S. ${ }^{2}$, Longo N. ${ }^{7}$, Martorana G. ${ }^{8}$, Novara G. ${ }^{6}$, Porpiglia F. ${ }^{9}$, Rocco F. ${ }^{10}$, Rovereto B. ${ }^{11}$, Schiavina R. ${ }^{8}$, Serni S. ${ }^{2}$, Simeone C. ${ }^{3}$, Volpe A. ${ }^{12}$, Carini M. ${ }^{2}$

${ }^{1}$ Azienda Ospedaliera Universitaria Federico II, Dept. of Urology, Naples, Italy, ${ }^{2}$ University of Florence, Dept. of Urology, Florence, Italy, ${ }^{3}$ University of Brescia, Dept. of Urology, Brescia, Italy, ${ }^{4}$ University of Modena, Dept. of Urology, Modena, Italy, ${ }^{5}$ Niguarda Hospital University of Pavia, Dept. of Urology, Pavia, Italy, ${ }^{6}$ University of Padua, Dept. of Urology, Padua, Italy, ${ }^{7}$ University Federico II of Naples, Dept. of Urology, Naples, Italy, ${ }^{8}$ University of Bologna, Dept. of Urology, Bologna, Italy, ${ }^{9}$ University of Turin, Dept. of Urology, Turin, Italy, ${ }^{10}$ University of Milan, Dept. of Urology, Milan, Italy, ${ }^{11}$ University of Pavia, Dept. of Urology, Pavia, Italy, ${ }^{12}$ University of Eastern Piedmont, Novara, Dept. of Urology, Novara, Italy

INTRODUCTION \& OBJECTIVES: Simple enucleation (SN) has similar oncologic outcomes compared to standard partial nephrectomy (SPN). To date there is no evidence of differences between the two techniques in terms of surgical results. The pourpose of this study is to compare intraoperative, early post-operative and pathological outcomes of SE and SPN in a prospective multicenter dataset.

MATERIAL \& METHODS: Patients treated with NSS for clinical T1 renal tumors between January 2009 and January 2011 were evaluated. The SPN and SE groups were compared regarding clinical, surgical, and pathologic outcome variables. Multivariable logistic regression models were applied to analyse predictors of warm ischemia time (WIT) $>20$ minutes and positive surgical margin (PSM) status.

RESULTS: Out of 535 patients included, 226 patients underwent SE and 309 SPN. SE was associated with longer WIT (18.3 min vs $16.2 \mathrm{~min}, \mathrm{p}=0.0022$ ), lower intraoperative blood loss (180 cc vs $248 \mathrm{cc}, \mathrm{p}=0.0084$ ) and shorter operative time (122 min vs $151 \mathrm{~min}$; p20 minutes. The incidence of PSM was lower in patients treated with SE $(1.3 \%$ vs $6.7 \% ; p=0.01$ ). At multivariate analysis, PSM was related to the surgical technique, with a 5 -fold increased risk of PSM for SPN compared to SE. The incidence of overall and medical complications was significantly higher in the SPN group.

CONCLUSIONS: SE is associated with better intraoperative and post-operative outcomes in terms of mean blood loss, mean operative times, incidence of PSM, overall and medical complications. Although WIT is longer during SE, the mean WIT remains under 20 minutes and the surgical technique is not an independent predictor of WIT > 20 minutes. 